International Journal of Pharmaceutics

Elsevier Editorial system(tm) for

Manuscript Draft

Manuscript Number: IJP-D-16-02261R1

Title: Apoferritin nanocage as streptomycin drug reservoir: technological optimization of a new drug delivery system

Article Type: Research Paper

Section/Category: Pharmaceutical Nanotechnology

Keywords: Apoferritin, encapsulation efficiency, nanocage, purification process

Corresponding Author: Professor Giovanni Tosi, PhD

Corresponding Author's Institution: University of Modena and Reggio Emilia

First Author: Barbara Ruozi, Associate Professor

Order of Authors: Barbara Ruozi, Associate Professor; Patrizia Veratti, PhD; Maria Angela Vandelli, Full Professor; Andrea Tombesi, PhD; Massimo Tonelli, PhD; Flavio Forni, Full Professor; Francesca Pederzoli, PhD; Giovanni Tosi, PhD

Abstract: The aim of this study is to formulate and characterize streptomycin-loaded apoferritin nanoparticles (ApoStrep NPs) for their potential therapeutic use in bacterial resistant infections (i.e. tuberculosis). Apostrep NPs were prepared by disassembly/reassembly process via pH method and changing apoferritin/drug molar ratio, purified by dialyses process also associated with gel filtration chromatography and characterized in their chemico-physical and technological parameters as yield, size distribution, polidispersivity, morphology, internal structure, zeta potential and loading efficacy. The results showed that spherical reproducible NPs could be obtained by using apoferritin/drug molar ratio lower than $1: 25$ and purification based on the combination of dialysis and gel filtration chromatography. Photon correlation spectroscopy, Uv-visible detection and electron microscopy showed the maintenance of the native apoferritin chemico-physical properties and structure. When formulated with apoferritin/drug 1:10 and 1:25 molar ratio, Apostrep NPs showed remarkable encapsulation efficacy (35\% and 28\%, respectively) along with kinetic profile of drug delivery, approximately $15 \%$ at $37{ }^{\circ} \mathrm{C}$ in $72 \mathrm{~h}$, as evidenced by "in vitro" release experiments. 
Dear Editor,

Please find enclosed our revised paper entitled "Apoferritin nanocage as streptomycin drug reservoir: technological optimization of a new drug delivery system "

We all hope that this paper version will answer to referee suggestions and we are confident that this version is now improved.

Please find uploaded the file describing the referee suggestions and in red highlighted our answer.

Within the revised text, we also highlighted any changes in red.

I thank you very much for your attention and please receive my warmest regards,

Giovanni Tosi

Associate Professor

Department of Life Sciences

University of Modena and Reggio Emilia

Italy 


\section{IJP AUTHOR CHECKLIST}

Dear Author,

It frequently happens that on receipt of an article for publication, we find that certain elements of the manuscript, or related information, is missing. This is regrettable of course since it means there will be a delay in processing the article while we obtain the missing details.

In order to avoid such delays in the publication of your article, if accepted, could you please run through the list of items below and make sure you have completed the items.

\section{Overall Manuscript Details}

- Is this the final revised version?

- Are all text pages present?

- Are the corresponding author's postal address, telephone and fax numbers complete on the manuscript?

- Have you provided the corresponding author's e-mail address? X

- Manuscript type - please check one of the following:

Full-length article

Review article

Rapid Communication

Note

Letter to the Editor

Other

- Manuscript section - paper to be published in:

Pharmaceutical Nanotechnology section

Personalised Medicine section

\section{Manuscript elements}

- Short summary/abstract enclosed? X

- 3-6 Keywords enclosed? X

- Complete reference list enclosed? X

- Is the reference list in the correct journal style? $\mathrm{X}$

- Are all references cited in the text present in the reference list? $\mathrm{X}$

- Are all original figures cited in the text enclosed? X

Electronic artwork format?

- Are figure legends supplied?

- Are all figures numbered and orientation provided?

- Are any figures to be printed in colour?

If yes, please list which figures here:-

- If applicable, are you prepared to pay for reproduction in colour?

- Are all tables cited in the text supplied?

\section{General}

- Can you accept pdf proofs sent via e-mail? 
Referee response

Reviewers' comments:

Reviewer \#1:

The paper by Ruozi et al deal with formulation and characterization of streptomycin-loaded apoferritin nanoparticles. The paper is well executed and written. The data could be useful in translational application in particular that of microbiology and infective diseases.

The findings should be of great interest to International Journal of Pharmaceutics readership. This is a solid piece of work that should be acceptable in the present form.

Reviewer \#2: The presented manuscript by Ruozi et al., with the title "Apoferritin nanocage as streptomycin drug reservoir: technological optimization of a new drug drug delivery systems" deals with the characterisation of novel apo-transferrin protein based particles, loaded with streptomycin, and explores optimal conditions for formulation and production. The authors test several apo-ferritin/streptomycin ratios and investigate features such as, among others, encapsulation efficacy, release kinetics, morphology, and size distribution. The study covers an interesting topic, the experiments are well designed and performed and the manuscript is well written. I therefore have only minor points that should be addressed before publication.

Specific comments:

1. The Material and Methods sections could benefit from more details and should be revised. Some examples: under 2. the authors state "After gently pipetting, apoferritin solution, monitored in term of size, surface charge and morphology (data not shown), was ..." How was this done exactly? Further, "...water, previously acidified to $\mathrm{pH} 2$, ..." How was this done?

Thanks, we corrected and ameliorated.

It would help the reader to (maybe in a table) provide the following information: In $3 \mathrm{~b}, 1 \mathrm{~mL}$ final apo-ferritin solution is obtained and further characterised. For the experiments used.

What is the concentration (molarity) of streptomycin in the solution, what is the concentration encapsulated into nanocages, what is the concentration of apo-ferritin in the solution and the concentration of formed nanocages. The information is present in the manuscript but should be summarized.

We corrected table I, in which there are yet information on encapsulation efficiency. Streptomycin in solution is concentrated at $0.05 \mathrm{mg} / \mathrm{mL}$.

The Streptomycin concentration is expressed on content, as reported in table 1.

So that, depending on the sample, as below:

\begin{tabular}{|l|l|}
\hline Samples & $\begin{array}{l}\text { mg Strep/100 } \\
\text { mg of Apo }\end{array}$ \\
\hline ApoStrep 1:10-D & $0.67 \pm 0.01$ \\
\hline ApoStrep 1:10- & $0.53 \pm 0.01$ \\
\hline
\end{tabular}




\begin{tabular}{|l|l|}
\hline DG & \\
\hline ApoStrep 1:25-D & $1.27 \pm 0.02$ \\
\hline $\begin{array}{l}\text { ApoStrep 1:25- } \\
\text { DG }\end{array}$ & $1.07 \pm 0.01$ \\
\hline
\end{tabular}

We added a table with the requested information.

\begin{tabular}{|l|l|l|}
\hline Sample Name & $\begin{array}{l}\text { Streptomycin concentration } \\
(\mathrm{mg} / \mathrm{mL})\end{array}$ & Apoferritin amount $(\mathrm{mg} / \mathrm{mL})$ \\
\hline Streptomycin free solution & $0.05 \mathrm{mg} / \mathrm{mL}$ & $\backslash$ \\
\hline Apoferritin solution & $\backslash$ & $15 \mathrm{mg} / \mathrm{mL}$ \\
\hline ApoStrep 1:10-DG & See table I & $10,3( \pm 0,2) \mathrm{mg} / \mathrm{mL}$ \\
\hline ApoStrep 1:25-DG & See table I & $10,1( \pm 0,3) \mathrm{mg} / \mathrm{mL}$ \\
\hline ApoStrep 1:50-DG & See table I & $2,9( \pm 0,2) \mathrm{mg} / \mathrm{mL}$ \\
\hline & & \\
\hline
\end{tabular}

Table II: concentration of Apoferritin and Streptomycin. For Streptomycin content, please see Table I.

2. The statistical evaluation needs a revision. Pairwise comparisons using t-test as stated in the Methods section is not appropriate. The evaluation should be done with ANOVA and in case of significant differences a post-hoc analysis such as Bonferroni.

We repeated the statistical analysis using the suggested methods.

3. In the results section, regarding the data shown in Figure 4 (page 136) the authors state a significant change in Zeta potential values. However, in the figure, no significance is indicated.

Yes, we missed to insert the significance which is now inserted.

4. In figure 3, it is hard to understand what comparisons are significant and marked by the "*" . Comparisons have to be done by ANOVA (see above) and significances labeled newly according to the new results.

We tried to allow a more easy reading of significance in the new version of the figure.

5. For the data presented in figure 7, can the authors extrapolate, when a full release would be achieved or perform the measurements for longer periods as the complexes seem quite stable.

Yes, we agree with the referee, we can estrapolate the timing of a full release, and also we tested over time the release. We got a very a long period of release, and we agree with the referee, the complexes are quite stable and remain, as they are or slowing releasing Streptomycin for a long period.

But since it is a formulative study, we are also aware that in vivo or even in vitro, this release would be affected by many factors which could strongly impact on the release $(\mathrm{pH}$, enzymes, proteins) and therefore we decided to stop at 72 hrs only to show this kind of stability within 3 days. 
6. The conclusion section is rather short. I suggest to add a paragraph discussing the usability of the novel system in clinics. For example, what is the concentration of streptomycin used for treatment in the clinics and can the concentration be reached by apo-ferrtin nanocage delivery? How many injections are necessary? Does the slow release rate present an advantage or disadvantage? What is the amount of apo-ferrtitin nanocages necessary for a treatment of a patient and is the production feasible in this amount?

We agree, we added a paragraph for the application of these system in clinics.

\section{Application of Apoferritin nanocages}

Despite extensive promises, much work is needed before clinical translation of APO as nanocages. As example, the study of the architecture and the structure of loaded APO are still at early stages and this situation represents the major limitation in translatability of APO to become DDS. This fact is principally due to unclear protocols for APO formulation and unclear chemico-physical, morphological and technological characterization and it frequently generates conflicting outputs. Regarding Streptomycin application, the dose normally used in therapy is very high, and it will be impossible to reach such dose with APO nanocages, but on the other hand these systems are not designed for this scope. In fact, the possibility of exploiting nanocages able to be "safe" when circulating in the bloodstream, to slowly release the loaded drug and in a controlled manner and most of all to be engineered for a selective delivery should be considered as an added value and the real potential of these systems. In this work, we also tried to answer to the general lack of characterization of these system, as we firstly analyzed the possibility of formulating an active agent different from the "commonly used" agents for loading into APO (namely anticancer drugs). Moreover, we moved on analyzing the nature and narrow size of APO complex using analytical technique commonly used to characterize synthetic DDS as liposomes or nanoparticles (TEM, AFM, SEM, PCS, DSC etc) and applying these technologies to APO cages characterization. Aiming to improve the chances of translatability of APO as drug delivery systems, another point, which is very hard to be investigated, is related to the drug release and in particular to the changes in morphology and architecture of APO cage during and after the release of the drugs. To our knowledge, the technologies for this kind of characterization are still lacking, even if this focus strongly affects the biocompatibility of APO and the efficacy of treatment promoted by the released drug. A huge work should be done to better investigate on these aspects that need to be considered with a major interest.

7. Carefully revise the manuscript for spelling and grammar. There are several errors and typos to be found throughout the manuscript. Fro example:

- I suggest to change the title to "Apoferritin nanocage as streptomycin drug reservoir: technological optimization of a new drug delivery system"

We agree with the referee and changed the title accordingly.

- page 130, Introduction: please make sure to have no space between a number and \% $(75 \%)$

Done.

-page 131, I. $1 / 2 \ldots$ to efficiently load drugs into $\ldots$

corrected

- page 136, 3rd paragraph "slightly"

corrected 
- page 138 "be set to stabilize and modulate drug release, ..." corrected 


\section{Disassembly/Reassembly protocol}

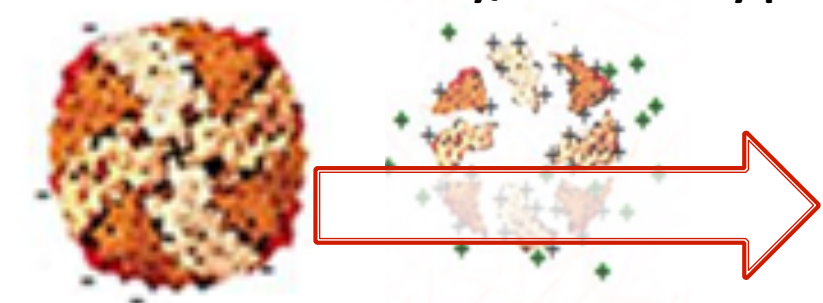

1:10- 1:25 -1:50 apoferritin/ drug molar ratio

\section{Purification}

(dialysis + gel filtration

chromatography)

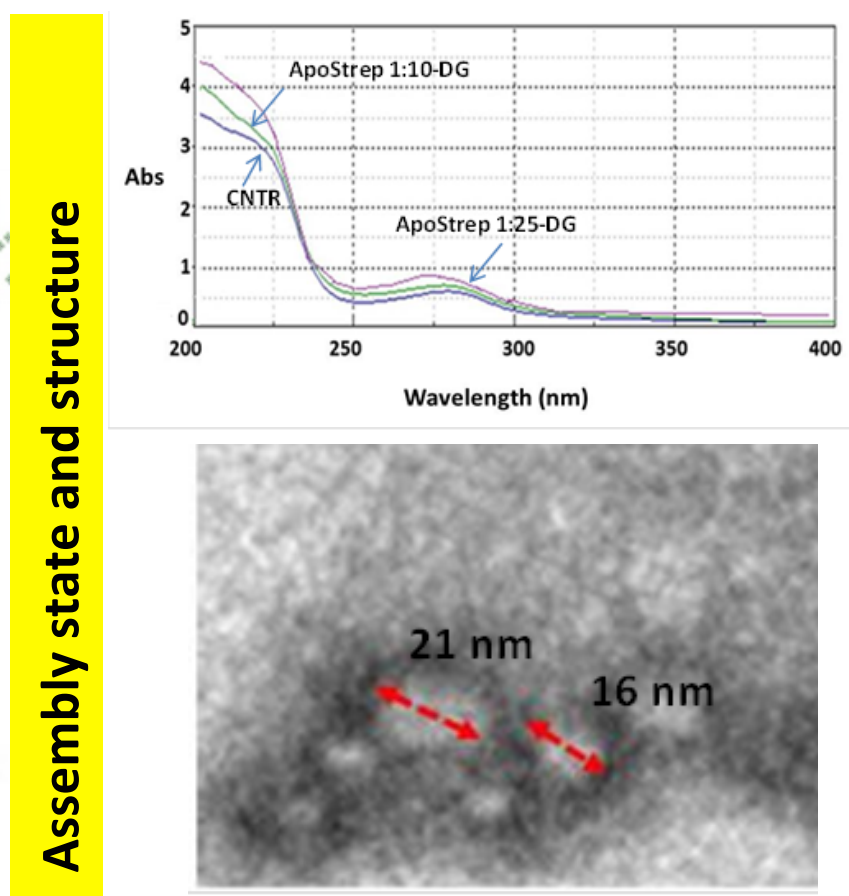

Chemico-physical and technological properties

\begin{tabular}{|l|l|c|c|c|l|l|}
\hline Samples & PDI & $\begin{array}{l}\mathrm{Di}(\mathbf{5 0}) \\
(\mathbf{n m} \pm \text { SD })\end{array}$ & $\begin{array}{l}\mathrm{Dn}(\mathbf{5 0}) \\
(\mathbf{n m} \pm \text { SD) }\end{array}$ & $\begin{array}{l}\text { Zeta pot } \\
(\mathbf{m v} \pm \text { SD })\end{array}$ & $\begin{array}{l}\mathrm{mg} / 100 \\
\mathbf{m g} \text { of apo }\end{array}$ & EE\% \\
\hline $\begin{array}{l}\text { Apoferritin } \\
\text { free/CNTR }\end{array}$ & $0.29 \pm 0.02$ & $40 \pm 0.5$ & $12 \pm 0.5$ & $-48 \pm 2$ & & \\
\hline $\begin{array}{l}\text { ApoStrep } \\
1: 10-D G\end{array}$ & $0.30 \pm 0.02$ & $25 \pm 2$ & $10 \pm 0.5$ & $-42 \pm 3$ & $0.53 \pm 0.01$ & $35 \pm 4$ \\
\hline $\begin{array}{l}\text { ApoStrep } \\
1: 25-D G\end{array}$ & $0.35 \pm 0.03$ & $20 \pm 1$ & $9 \pm 1$ & $-41 \pm 4$ & $1.07 \pm 0.01$ & $28 \pm 1$ \\
\hline
\end{tabular}

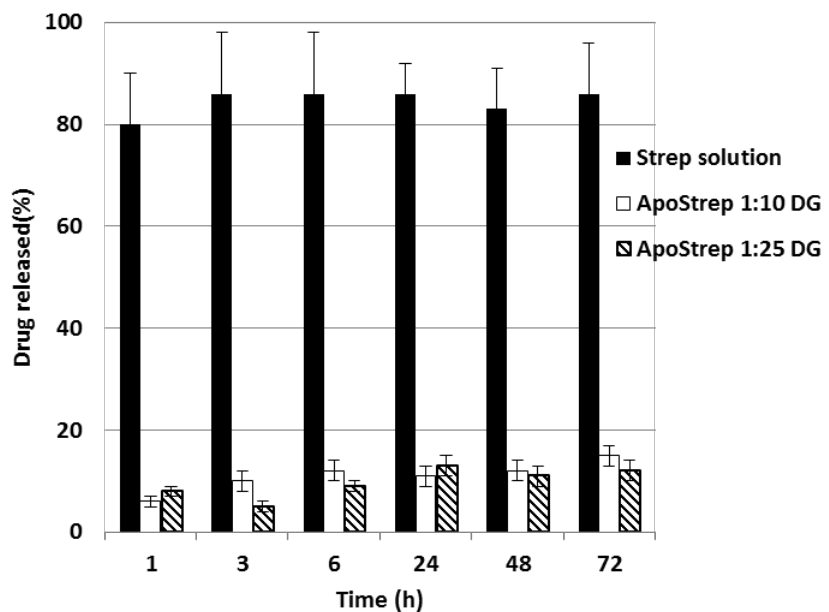




\title{
Apoferritin nanocage as streptomycin drug reservoir: technological optimization of a new drug delivery system
}

\author{
B. Ruozi ${ }^{1}$, P. Veratti ${ }^{1}$, M.A. Vandelli ${ }^{1}$, A. Tombesi ${ }^{2}, M$. Tonelli $^{2}$, F. Forni ${ }^{1}$, F. Pederzoli $^{1}$, D. Belletti ${ }^{1}$, G. \\ Tosi $^{1^{*}}$ \\ ${ }^{1}$ Department of Life Sciences, Via Campi 103, University of Modena and Reggio Emilia, 41124 \\ Modena- ${ }^{2} \mathrm{CIGS}$, Centro Interdipartimentale Grandi Strumenti, University of Modena and Reggio \\ Emilia, Italy
}

*Corresponding author: Prof. Giovanni Tosi, Department of Life Sciences, phone: +390592058563 , email: gtosi@unimore.it

\section{Abstract}

The aim of this study is to formulate and characterize streptomycin-loaded apoferritin nanoparticles (ApoStrep NPs) for their potential therapeutic use in bacterial resistant infections (i.e. tuberculosis). ApoStrep NPs were prepared by disassembly/reassembly process via $\mathrm{pH}$ method and changing apoferritin/drug molar ratio, purified by dialyses process also associated with gel filtration chromatography and characterized in their chemico-physical and technological parameters as yield, size distribution, polidispersivity, morphology, internal structure, zeta potential and loading efficacy. The results showed that spherical reproducible NPs could be obtained by using apoferritin/drug molar ratio lower than 1:25 and purification based on the combination of dialysis and gel filtration chromatography. Photon correlation spectroscopy, Uv-visible detection and electron microscopy showed the maintenance of the native apoferritin chemico-physical properties and structure. When formulated with apoferritin/drug 1:10 and 1:25 molar ratio, ApoStrep NPs showed remarkable encapsulation efficacy ( $35 \%$ and $28 \%$, respectively) along with kinetic profile of drug delivery, approximately $15 \%$ at $37^{\circ} \mathrm{C}$ in $72 \mathrm{~h}$, as evidenced by "in vitro" release experiments.

\section{Introduction}

Streptomycin is a broad spectrum aminoglycoside antibiotic typically used for the treatment of active tuberculosis, in combination with other antituberculosis agents. It is composed of a metabolic product of Streptomyces globisporus or another similar organism (Zumla et al., 2013). The major disadvantages of streptomycin consists of the inadequate penetration into the cells (due to its hydrophilicity) and rapid elimination from the blood stream (due to both efficient renal filtration and low level of association to plasma proteins) (Coessens et al., 1996). Moreover, different evidences describe a rapid bacterial resistance oocurring after Streptomycin treatment (75\% of bacterial infectious including tubercoloses after one-four months of treatment). In most cases, this resistance has proven to be permanent. The importance of this problem is obvious, since further treatment with streptomycin were shown to be ineffective when the infecting organisms are drug-resistant (Pfuetze 1948). Above the development of novel antitubercolosis drugs, the creation of nanocarriers able to modulate drug delivery could be interestingly applied on drug-resistant epidemic. In fact, loading currently-used antitubercolosis drugs in nanocarriers-based formulations may shorten drug regimen duration, reduce frequency, and deliver medications more efficaciously. That way, a sustained release in both blood plasma as well as organ tissue, an increase in bioavailability, the reduction of patient default and the improvement of completion rates could be achieved. Taken together, all these advantages could lead to significant potential in the reduction of drug resistant tubercolosis cases (Smith 2011; Asadi 2014). 
An exciting improvement in drug delivery systems is represented by the possibility to efficiently load drugs into apoferritin nanocage. Apoferritin is the demineralized ferritin, composed of 24 polypeptide subunits packed together to form a nanosphere of diameter $\sim 12 \mathrm{~nm}$ with an internal cavity $\sim 8 \mathrm{~nm}$. This protein, as drug delivery system, was demonstrated to be a promising vehicle for targeted delivery of anticancer drugs (Arosio et al., 1991, Belletti et al., 2016). Generally, apoferritin may enhance drug selectivity for cell surfaces that express ferritin receptors, in particular ferritinbinding sites and endocytotic pathways, which are expressed in all body cells and mainly in cells in rapid proliferation (Burdo and Connor, 2003; Aisen 1991). Besides these aspects, apoferritin displays a number of advantages which could be exploited in the application toward drug-resistant tubercolosis epidemic. Ferritin is a major iron storage protein in humans; therefore its biocompatibility and biosafety are assured. Moreover, compared with other drug carriers (i.e. polymeric nanoparticles and liposomes), apoferritin is much smaller in size, thus leading to a longer circulation half-life. Despite the rigidness under physiological conditions, the ferritin nanocages can be broken down into subunits in acidic environment by a reversible process. In fact, when the $\mathrm{pH}$ is tuned back to neutral, the subunits are reconstituted into nanocage structures, almost in an intact manner (Maham et al., 2009; Lin et al. 2011). Another advantage of apoferritin consists of its chemical "reactivity" since, as polymeric nanoparticles or liposomes, its surface can be easily modified to selectively direct to target site (Lin et al., 2011; Uchida et al., 2006; Dominguez-Vera 2004).

Regarding the drug to be loaded into apoferritin, streptomycin (and particularly sulfate salt used in this research) possesses chemico-physical properties favorable for its location into the apoferritin nanocage, as its stability both in acids and in bases which is needed for formulative protocols requiring extreme $\mathrm{pH}$ values. Streptomycin is featured by a pKa of about 8 due to its secondary amino group and pKa ranging between 10 and 12 due to its two guanidine groups (Nys et al., 1971), providing a net positive charge both in physiological envirorment $(\mathrm{pH} 7)$ and during the formulation conditions (acidic $\mathrm{pH}$ ), which favors electrostatic interaction with the apoferritin. Moreover, the MW (723 Da) is suitable for a stable internalization into protein cage avoiding drug loss across protein pores.

In this paper, starting from preformulative evaluation on several parameters involved in disassembly/reassembly protocols (protein concentration, volume of reaction, $\mathrm{pH}$ during the process, ionic concentration in solution, temperature, stirring), we aimed to optimize the technological conditions needed for stable formulation of streptomicyn into apoferritin nanoparticles (ApoStrep NPs). Particularly, we focused on the impact of the molar ratio between apoferritin and drug (1:10, $1: 25,1: 50$ ) and the purification process (by comparing dialyses and gel filtration chromatography) on drug loading, without changing the native structure of ApoStrep NPs. Then, we tried to consolidate the possibility of using apoferritin as drug delivery systems, not only for anticancer drugs (Belletti et al., 2016), but also for other kinds of drugs and active molecules.

\section{Materials and Methods}

\section{Chemicals}

Apoferritin from equine spleen (solution $\sim 40 \mathrm{mg} / \mathrm{mL}$ in $0.15 \mathrm{M}$ sodium chloride) and streptomycin sulfate (MW $=728.69$, water solubility $=50 \mathrm{mg} / \mathrm{mL}$ ) were purchased from Sigma Aldrich (Milan, Italy). Acetate cellulose dialysis membranes were purchased from CelluSepT1 (MWCO:3500, Membrane Filtration Products, Seguin, TX, USA). Sepharose CL-2B resin was purchased from Sigma Aldrich (Milan, Italy). Micro BCA protein assay kit composed of reagent A (alkaline tartrate-carbonate buffer), reagent $B$ (bicinchonic acid solution) and reagent $C$ (copper sulfate solution) was purcheased from Thermo Fisher Scientific Inc (Milan, Italy). All the solvents were of analytical grade; all other chemicals were commercially obtained and used without further purification. A MilliQ water system (Millipore, Bedford, MA, USA), supplied with distilled water, provided high-purity water (18 M $\Omega$ ). 


\section{The encapsulation of streptomicine in apoferritin via $\mathrm{pH}$ changing method}

Streptomicyin was formulated in apoferritin NPs by disassembly/reassembly protocols accordingly with others adopted for different types of drugs (Xing et al., 2009; Cutrin et al., 2013; DominguezVera 2004; Blazkova et al., 2013; Liu et al., 2006; Kilic et al., 2012). For all preparations, apoferritin solution (15 mg/mL, $0.031 \mu$ moles) was obtained by diluting apoferritin stock solution with water (370 $\mu \mathrm{L}$ diluted to $1 \mathrm{~mL}$ ). After gently pipetting, apoferritin solution, monitored in term of size and surface charge (by means of PCS using a Zetasizer Nano ZS, Malvern UK as explained in paragraph 4a) and morphology (by means of trasmission electron microscopy (TEM) analysis as explained in paragraph 4b), was acidified to $\mathrm{pH} 2$ (by addition of $800 \mu \mathrm{L}$ of $0.1 \mathrm{~N} \mathrm{HCl}$ ), thus providing the disassociation of the apoferritin subunits. The sample was mixed by using magnetic stirring for 20 $\mathrm{min}$ at $20^{\circ} \mathrm{C}$. Then, different amounts of streptomycin sulfate $(0.23,0.57,1.14 \mathrm{mg}$ corresponding to $0.31,0.78$ and $1.56 \mu$ moli, respectivelly) solubilized in water $(200 \mu \mathrm{L})$ and acidified to the $\mathrm{pH} 2$ (addition of $\mathrm{HCl} 0.1 \mathrm{~N}$ ) was added dropwise to the protein solution to finally have 1:10, 1:25, 1:50 apoferritin/drug molar ratios. As control sample, un-loaded apoferritin NPs were obtained following the same protocol previously exposed. Briefly, $200 \mu \mathrm{L}$ of water, previously acidified to $\mathrm{pH} 2$ (by addition of $0.1 \mathrm{~N} \mathrm{HCl}$ ), was added to apoferritin solution $(\mathrm{pH} 2)$, simulating the same increase in volume of the case of addition of drug solution.

In order to recover the native structure of the protein, the $\mathrm{pH}$ value of the solution was adjusted to $\mathrm{pH} 8$ by adding $\mathrm{NaOH} 0.1 \mathrm{~N}$ (about $700 \mathrm{uL}$, under magnetic stirring). The final volume was adjusted to $3 \mathrm{~mL}$ by adding water (basified to $\mathrm{pH}$ 8). The resulting solution was then stirred over $2 \mathrm{~h}$ at $25^{\circ} \mathrm{C}$ to allow the complete structural reassembly of the protein.

The following formulations were obtained: CNTR (unloaded), ApoStrep 1:10, ApoStrep 1:25 and ApoStrep 1:50 (streptomicyn loaded NPs).

\section{Purification of samples}

\section{3a. Dialysis procedure}

A membrane diffusion method was used to purify free streptomycin from streptomycin loaded NPs. Each sample ( $1 \mathrm{~mL}$ of suspension) was placed in the dialysis tube (MWCO $3500 \mathrm{Da}$ ), permeable to drug alone, but not to NPs. Tube was closed at both ends using closure devices (Spectrum Medical

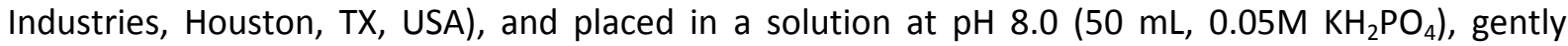
shaken with a magnetic stirrer (100 rpm at $20 \pm 1^{\circ} \mathrm{C}$ for $4 \mathrm{~h}$ ). Free drug diffused out of samples and through the membrane to the receiver phase. After this time, volume of receiving phase was then adjusted to $1 \mathrm{~mL}$ by using rotatory evaporation, filtered by a acetate cellulose (AC) membrane with $0.2 \mu \mathrm{m}$ porosity (Sartorious, Göttingen, Germany).

Samples purified by dialysis were recovered and named: CNTR-D, ApoStrep 1:10-D, ApoStrep 1:25-D and ApoStrep 1:50-D.

\section{3b. Gel filtration chromatography}

The apoferritin samples, recovered after dialysis purification and adjusted to $1 \mathrm{~mL}$ as final volume, was eluted through the Sepharose CL-2B resin (Sigma Aldrich), packed in $17.5 \times 1.5 \mathrm{~cm}$ column, by using $0.05 \mathrm{M} \mathrm{KH}_{2} \mathrm{PO}_{4}$ solution ( $\mathrm{pH} 8.0$ ) as mobile phase. The separation efficiency was achieved by working in "flash mode", exerting a pressure of about 9 bar by means of an $\mathrm{N}_{2}$ flow. 40 fractions were collected, one of each minute starting from the $3^{\text {rd }}$ min after deposition. Fractions were analyzed by UV spectrophotometry (Jasco V-530 UV-VIS Spectrophotometer, JASCO Europe, Cremella, Italy) by means of spectral scanning acquisition mode over the wavelength range $200-400 \mathrm{~nm}$, with $5 \mathrm{~min}$ measurement intervals. The collected fractions containing apoferritin (identified by typical peak at $280 \mathrm{~nm}$-UV absorbance) were submitted to test by PCS, which highlighted the elution of apoferritin NPs within the interval 8-15 min (data not shown). These fractions were mixed and the volume adjusted to $3 \mathrm{~mL}$ (by rotary evaporation). Purified Samples obtained were named CTRL-DG, ApoStrep 1:10 DG, ApoStrep 1:25 DG and ApoStrep 1:50 DG. 
Fraction from 3 to 8 min containing aggregates were eliminated, while fractions from 16 to $43 \mathrm{~min}$ were mixed, concentrated in volume $(1 \mathrm{~mL})$ and then analyzed by HPLC to evaluate the presence of free streptomycin.

\section{Chemico-physical characterization of apoferritin NPs}

\section{4a. Size, polydispersivity and zeta potential}

Polydispersivity index (PDI) and size distribution were determined by PCS using a Zetasizer Nano ZS (Malvern, UK; Laser $4 \mathrm{~mW}$ He-Ne, $633 \mathrm{~nm}$, Laser attenuator Automatic, transmission 100-0.0003\%, Detector Avalanche photodiode, Q.E. > 50\% at $633 \mathrm{~nm}$ ). The results were expressed both as intensity distribution, $D(i)$, based on the scattered light intensity and as number distribution, $D(n)$, based on the number of particles that peculiarly scattered light of all the particles. Both $D(i)$ and $D(n)$ identified the size below which is placed the $10 \%[D(10)], 50 \%[D(50)]$ and $90 \%[D(90)]$ of all the NPs. The zeta potential was measured using the same equipment with a combination of laser Doppler velocimetry and phase analysis light scattering (PALS). All the data are expressed as means of at least three determinations carried out for each preparation lot (three lots for each sample).

\section{4b. Morphology and structure}

The morphological and microstructural characterization of NPs was carried out through trasmission electron microscopy (TEM). A drop of each sample (CNTR-DG, ApoStrep 1:10 DG, ApoStrep 1:25 DG) was placed on a 200-mesh copper grid (TABB Laboratories Equipment, Berks, UK) and allowed to adsorb. The excess suspension was removed by filter paper, sample dried and then NPs were imaged with TEM, operating at an acceleration voltage of $200 \mathrm{kV}$ (model JEM 2010; JEOL, Peabody, MA). Structure of apoferritin forming the NPs (CNTR-DG, ApoStrep 1:10 DG, ApoStrep 1:25 DG) was also investigated by evaluating the UV-vis absorption spectra performed on a Jasco V-530 UV-VIS spectrometer supplied with Spectra Manager II software.

\section{Apoferritin recovered after purification}

BCA assays were performed to achieve apoferritin quantification in CNTR-DG, ApoStrep 1:10 DG, ApoStrep 1:25 DG and ApoStrep 1:50 DG samples. Assays were performed using 96-well microplates and Pierce BCA Protein Assay Kit (Reagents A, B, C) (Rodano, Milano, Italia). Sample and reagent preparations were executed as indicated by manufacturing instructions. Briefly, in each well, an exact amount $(150 \mu \mathrm{L}$ ) of samples (CNTR-DG, ApoStrep 1:10 DG, ApoStrep 1:25 DG or ApoStrep 1:50 DG) previously diluted $1: 25$ with $0.05 \mathrm{M} \mathrm{KH}_{2} \mathrm{PO}_{4}$ solution $(\mathrm{pH} 8)$ or standard solutions $(15 \mathrm{mg} / \mathrm{mL}$ of apoferritin solution) was added to the reagent mix $(150 \mu \mathrm{L})$. Microplate was stored at $37^{\circ} \mathrm{C}$ for $2 \mathrm{~h}$ to allow the bicinchoninic acid/ $\mathrm{Cu}^{2+}$ reaction with proteins, then the absorbance of the reaction product for the colorimetric quantification of proteins was measured at $570 \mathrm{~nm}$, subtracting the absorbance reading of the blank by means of Microplate Reader Multiscan (Spectrum Finstruments ${ }^{\circledR}$ ). The concentration was calculated using the calibration curve previously set up (linearity was assumed in the range of $0.46-50 \mu \mathrm{g} / \mathrm{mL}$ ).

Apoferrritin recovered after purification process/dialyses and gel filtration chromatography(ApoYield [\%]) is represented by the percent weight fraction of apoferritin evaluated by BCA assay compared with the total weight of apoferritin used for the preparation (Apo).

The yield [\%] was calculated as follows:

$$
\text { ApoYield }[\%]=\frac{A p o \text { from BCA test } x 100}{(\text { Apo })}
$$




\section{Technological characterization}

\section{6a. Encapsulation efficiency}

The encapsulation efficiency of streptomycin into apoferritin NPs was calculated by indirect evaluation by HPLC analysis of the drug diffused through the dialysis membrane or eluted by gel filtration chromatography. The HPLC apparatus (JASCO Europe) used for this analysis is composed of a Model PU2089 pump provided with an injection valve with a 100 uL sample loop (Model 7725i, Jasco) and the UV/VIS detector (Jasco UV-975 Intelligent UV-VIS Detector). The analysis was performed in isocratic conditions, at a flow rate of $1 \mathrm{~mL} / \mathrm{min}$ and at $30^{\circ} \mathrm{C}$ by reverse-phase chromatography (RP/HPLC) on C18 Acclaim Polar Advantage II column (150x46 mm, pore size $5 \mu \mathrm{m}$, Agilent Technologies) equipped with a security guard. The mobile phase was $\mathrm{KH}_{2} \mathrm{PO}_{4}$ solution $(\mathrm{pH} 8.5$, $0.05 \mathrm{M}$ ). Chromatographic peak area of the samples were recorded at $200 \mathrm{~nm}$ and analyzed using a JASCO software (JascoBorwin 1.5). The concentration was calculated using the calibration curve previously set up (linearity was assumed in the range of $0.5-83 \mu \mathrm{g} / \mathrm{mL}$ ). The amount of streptomycin loaded into NPs was evaluated by difference between the amount of total drug used and drug excluded by NPs. Then, the percentages of encapsulation efficiency (EE) was calculated using the following equations:

\section{Encapsulation Efficiency (EE\%) $=$ [drug loaded into apoferritin NPs / total amount of drug used to formulate] X 100.}

All the data are expressed as the means of at least three determinations.

\section{$\underline{6 b .}$ In vitro release studies}

A membrane diffusion method was also used to evaluate the in vitro release studies of drug from apoferritin NPs. Briefly, $1 \mathrm{~mL}$ of Streptomycin $(0.05 \mathrm{mg} / \mathrm{mL})$ or streptomicyn loaded NPs was placed into the dialysis tube (CelluSep, MWCO 3500). Dialysis tube was closed and placed in water $(10 \mathrm{~mL})$ and gently shaken with a magnetical stirrer in water $\left(100 \mathrm{rpm}\right.$ at $\left.37^{\circ} \mathrm{C} \pm 0.1^{\circ} \mathrm{C}\right)$. The drug diffused out of samples and through the membrane to the receiver phase. At defined time intervals $(1,3,6,24$, 48 and $72 \mathrm{~h}$ ), a fixed volume $(1 \mathrm{~mL})$ of the receiving phase was withdrawn, the volume reduced at $100 \mathrm{uL}$ by evaporation and analyzed by HPLC for the drug content. The receiving phase tested was then replaced into the receiving medium to keep constant the final volume. The release study was carried out in triplicate for each sample.

\section{Statistical analysis}

All data were expressed as averages with standard deviations (SD). Statistical analysis was carried out using GraphPad Prism ${ }^{\mathrm{TM}}$. Data were assessed using either one-way or two-way ANOVA with a Bonferroni post hoc test. The significance was set at $P<0.05$.

\section{$\underline{\text { Results }}$}

\section{Streptomycin encapsulation into the apoferritin nanocage}

Figure 1. A) Schematic illustration of the encapsulation of streptomycin into apoferritin nanocage by disassembly/reassembly process via " $\mathrm{pH}$ changing method". Turbidity observed from $\mathrm{pH} 4$ to 8 is possible due to a) protein precipitation (isoelectric point at $\mathrm{pH} 4.5$ ); b) protein aggregation induced by multivalent cations as streptomycin sulphate at $\mathrm{pH} 7.4$; c) drug that could impact on the correct protein reassembling. 
A schematic rappresentation of strepytomicine encapsulation into the apoferritin is shown in Figure 1. In order to entrap the drug molecules into the apoferritin cage, the protein was disassembled and reassembled into the nanostructure via a "pH-changing method" as described in methods. The experimental conditions and parameters (buffer composition, concentration, volume of reaction, time of stirring, final $\mathrm{pH}$ ) were chosen on the basis of both preliminar investigations on disassembly/reassembly process of apoferritin alone and on the peculiar properties of drug (i.e. stability in the range $\mathrm{pH} \mathrm{3-8} \mathrm{at} \mathrm{room} \mathrm{temperature).} \mathrm{In} \mathrm{detail,} \mathrm{apoferritin} \mathrm{and} \mathrm{streptomycin} \mathrm{were}$ dissolved in MilliQ water and acidified with a $\mathrm{HCl} 0.1 \mathrm{~N}$, not observing turbidity nor precipitation. During the reassembly process, by adding $\mathrm{NaOH} 0.1 \mathrm{~N}$, once over the isoelectric point of the protein $(\mathrm{pH} 4.5)$, apoferritin precipitation took place, with difficult recovery of the monomeric form. This phenomenon was detected in the presence of high drug concentrations and probably due to the strong electrostatic attraction between positive charges of streptomycin and negative charges of the protein over its isoelectric point.

Multivalent cations, such as streptomycin, could act as a bridge with protein molecules, thus reducing intermolecular repulsion and maybe causing aggregation (Weisenberg and Timasheff, 1970). Alternatively, this bridge streptomycin-apoferritin may also induce incorrect interaction between protein subunits leading to wrong or incomplete reassembly (Figure 1).

In order to reduce this protein instability, reassembly process must be continued until $\mathrm{pH} 8.0$, therefore not stopping basification at $\mathrm{pH}$ 7.4. At this $\mathrm{pH}$ value, the drug loses one of its positive charges, with consequent reduction of its pro-aggregating effect, even maintaining stability (Oswald and Nielsen, 1947).

Remarkably, this reassembling protocol was effective in avoiding protein precipitation only using protein/drug molar ratio lower than 1:50 ratio, while it was poorly efficacious doubling the amount of drug (data not shown).

The polydispersity index (PDI) of samples after disassembly/reassembly of apoferritin with drug at different molar ratio (ApoStrep 1:10, ApoStrep 1:25 and ApoStrep 1:50) is reported in Figure 2. PDI values bigger than 0.3 suggest poor uniformity of all samples before purification process. Already disassembling/reassembling process of apoferritin carried out without drug seems to produce a sample (CNTR) with a multimodal distribution, presumably formed by monomers and aggregates.

The heterogeneity of samples proportionally increased with the increase of streptomycin concentrations used in formulation. Dialysis process, which removes the excess of drug in solution, lead to the stabilization complexes (ApoStrep 1:10-D, ApoStrep 1:25-D) with PDI values similar to apoferritin alone ones (of about 0.4). On the contrary, dialysis process was ineffective on ApoStrep 1:50-D, which tended to aggregate with an increase in PDI value up to 0.8 . In order to more efficiently remove aggregates, all samples underwent to a double purification process by combining dyalises with gel filtration. For all samples (ApoStrep 1:10-DG, ApoStrep 1:25-DG, ApoStrep 1:50DG) PDI values decreased down to 0.3-0.35, indipendentetly from the protein:drug molar ratio used.

Figure 2. PDI values of apoferritin NPs (CNTR, ApoStrep 1:10, ApoStrep 1:25, ApoStrep 1:50) after $\checkmark$ formulation, $\square$ dialysis process $(D)$ and - combining dialysis and gel filtration chromatography.

Figure 3. Dimensional characterization of ApoStrep NPs. D(i) (panels $a, c$ and e) and $D(n)$ (panels $b, d$ and $f$ ) of samples after formulation ( $a$ and $b$ ), dialysis ( $c$ and $d$ ), and combining dialysis and gel filtration chromatography (e and f). Statistical analyses were performed: ${ }^{*} p<0.05$ vs CNTR.

As suggested by PDI values, ApoStrep NPs can be described by a multimodal distribution, with the presence of apoferritin NPs and aggregates. To describe the sample, both Di and Dn were analized. Di values, referred to scattering intensity, nicely identify the presence of aggregate since large particles 
scatter much more light than small particles. On the contrary, Dn values clearly indicate the numerical distribution of the populations into the samples and therefore the most representative NPs population within the sample (organization of monomeric form of protein).

During the reassembly process, even in absence of drug (CNTR, Figure 3a), the sample resulted composed by protein nanocage (correct reassembly, $\mathrm{Di}(50)=57 \pm 17 \mathrm{~nm}$ ) mixed with protein aggregates (uncorrect reassembly, $\mathrm{Di}(90)=305 \pm 46 \mathrm{~nm}$ ).

$\mathrm{Di}(50)$ values of ApoStrep samples (ApoStrep 1:10, ApoStrep 1:25 and ApoStrep 1:50) proportionally increased in line with the increase of Streptomycin in solution, suggesting that a limit in drug concentration is present in order to preserve the integrity of apoferritin. The formulation obtained with 1:10 protein/drug molar ratio (ApoStrep 1:10) showed a Di(50) of 53 $\pm 15 \mathrm{~nm}$ similar to the CNTR (without drug, $\mathrm{Di}(50)=57 \pm 17 \mathrm{~nm}$ ), while already 1:25 protein/drug molar ratio formulation (ApoStrep 1:25) slightly start aggregating $(\mathrm{Di}(50)=100 \pm 20 \mathrm{~nm})$. This aggregation effect becomes significant in 1:50 protein/drug molar ratio (ApoStrep 1:50), with an increase of $\mathrm{Di}(50)$ at $220 \pm 60 \mathrm{~nm}$ value (Figure 3a).

Similarly, high values of Di90 and large S.D., representative of the presence of aggregates in suspension, slightly increased with the increasing of the drug into the formulation becoming higher that $1 \mathrm{uM}$ and less reproducible at 1:50 protein/drug ratio.

The simple incubation of drug and apoferritin at $\mathrm{pH} 8$ is not able to induce aggregation (data not shown), confirming that the disassembly/reassembly process is responsible of the protein destabilization in presence of the drug.

Dn values (Figure $\mathbf{3 b}$ ), which are more representative of monomeric population of protein, are nicely in agreement with $\mathrm{Di}$ values. Thus, protein aggregation was detected only in presence of protein/drug 1:50 molar ratio (ApoStrep 1:50), while no significant differences in Dn values were observed for 1:10 (ApoStrep 1:10) and 1:25 (ApoStrep 1:25) protein/drug molar ratios with respect to the control preparation (CNTR), demonstrating structural stability of the formulated apoferritin.

Purification of the sample from the excess of unloaded streptomycin lead to reduction of aggregation, as highlighted by Di values decrease (Figure 3c). No substantial differences in terms of Dn values were observed after purification by dialysis of the samples 1:10 and 1:25 molar ratio between protein and drug (ApoStrep 1:10-D, ApoStrep 1:25-D), indicating that size distribution of the re-organized protein nanosctructure is poorly affected by the removal of the excess of drug or protein aggregates (Figure 3d). Differently, the formulation obtained by using drug in large excess (ApoStrep 1:50-D) was not efficiently purified, maintaining a wide dimensional range of structures wrongly re-organized after loading/reassembly process.

The removal of aggregates is obtained by coupling gel filtration chromatography to dialysis process. As evident in Figure $\mathbf{3 e}$, for all the samples tested, the values of $\mathrm{Di}$ indicate a monomodal size distribution, referred to monomeric and monodisperse form of apoferritin nanostructure. Particularly, Di (50) values for the samples 1:10 and 1:25 molar ratio between protein and drug (ApoStrep 1:10-DG, ApoStrep 1:25-DG) begin to correlate with Dn values reaching under $30 \mathrm{~nm}$.

Figure 4 shows zeta potential values of apoferritin NPs obtained with different protein/drug molar ratio before and after the purification process. Regarding control sample (CNTR), high negative values of zeta potential on the surface on apoferritin $(-50 \pm 6 \mathrm{mV})$ confirmed high stability before and after the purification process. Zeta potential values significantly changed after the encapsulation process becoming less negative for all the molar ratio of tested complexes (from -20 to $-37 \mathrm{mV}$ ) if compared to apoferritin alone. This fact is probably due to a relevant absorption of streptomycin on NPs surface. This data was confirmed by the restoring of more negative zeta values (ranging from -38 $\mathrm{mV}$ and $-55 \mathrm{mV}$ ) after removal of excess of drug molecules by dialysis. The combination of dialysis and gel filtration chromatography did not change those negative values observed after the purification with dialysis process alone.

Figure 4. Zeta potential of apoferritin NPs (CNTR, ApoStrep 1:10, ApoStrep 1:25, ApoStrep 1:50) after formulation, $\square$ dialysis process $(D)$ and combining dialysis and gel filtration chromatography. 
As previously exposed, PCS analysis showed several concerns on formulations prepared with 1:50 apoferritin/drug molar ratio, strongly affected by the presence of aggregates. The amount of protein evaluated by BCA assay in apoferritin NPs fraction eluted from column by gel filtration chromatography confirms the suitability of this sample. In fact, apoferritin in ApoStrep 1:50-DG sample was only around $20 \%$ of Apo ferritin used in formulation, while apoferritin recovered in ApoStrep 1:10-DG and ApoStrep 1:25-DG was higher and close to $70 \%$ for both samples (Table II). Therefore, ApoStrep 1:50-DG was excluded from the subsequent experiments.

Table II: concentration of Apoferritin and Streptomycin. For Streptomycin content, please see Table I.

In order to examine whether the structure of apoferritin changed after the encapsulation of streptomycin (Xing et al., 2009), we performed and compared the UV spectra of the CNTR-DG with ApoStrep1:10-DG and ApoStrep1:25-DG (Figure 5). Apoferritin alone and ApoStrep NPs exhibited similar spectral features with their characteristic absorption (close to $280 \mathrm{~nm}$ ). This absorption arises from the amino acid residues with aromatic rings in apoferritin and its intensity linearly correlates with the concentration of protein (Stoscheck 1990). The results suggested that apoferritin drug complexes displayed structures similar to apoferritin alone and that encapsulation of streptomycin in the protein cavity barely affects the surface nature of apoferritin.

\section{Figure 5. UV spectra of CNTR-DG vs ApoStrep1:10-DG and ApoStrep1:25-DG}

The assembly state of the ApoStrep NPs was investigated using TEM analyses. TEM images revealed that disassembly/reassembly process, carried out both with or without drug, produces constructs that retain the native ability to adopt a spherical cage-like structure. As shown in Figure 6a, related to sample CNTR-DG (obtained without drug and purified by dialyses and gel filtration chromatograpy), the absence of electron-spot in correspondence of the central cavity of the particles, typical of TEM analysis of ferritin (Massover 1993), and the elemental analysis performed on the samples demonstrates that the protein is free of iron normally located in its internal cavity, making the core available for a subsequent load of drugs. A much more contrasting corona that surrounds the protein may in fact identifiy the densest area of the protein wall, while the lighter interior area could indicate its cavity. No differences both in morphology and size were observed between CNTR-DG and streptomicine loaded samples by using TEM experiments (Figure $\mathbf{6 c}$ and $\mathbf{d}$, as example we report only ApoStrep 1:25-DG).

Figure 6. TEM analysis of apoferritin NPS a) images of CNTR-DG, b) EDX of CNTR-DG, c) images of ApoStrep 1:25-DG, d) magnification of ApoStrep 1:25-DG.

\section{Encapsulation Efficiency and in vitro drug release}

The quantification of the encapsulated streptomycin was performed by indirect evaluation of the unloaded drug, separated from ApoStrep NPs both after dialysis process and the combination of dialysis and gel filtration chromatography. Results are reported in Table 1. The amount of drug encapsulated in $100 \mathrm{mg}$ of apoferritin is around $0.6 \mathrm{mg}$ and $1.3 \mathrm{mg}$ for ApoStrep1:10-D and ApoStrep 1:25-D, respectively. Combining gel filtration chromatography, the amount of drug stably encapsulated slightly decreased to $0.5 \mathrm{mg}$ and $1.0 \mathrm{mg}$, respectively. The encapsulation efficiency ranged from 30 to 40\% in ApoStrep1:10-DG, while it is closed to 30\% in ApoStrep1:25-DG, therefore confirming that the dialysis process is yet efficacious to purify the samples.

Moreover, apoferritin exhibited a good control on streptomycin release; in fact, independently of samples tested, only $15 \%$ of encapsulated drug was released from protein nanocage over three days at $37^{\circ} \mathrm{C}$, demonstrating higher stability of formulate products (igigure $\mathbf{7}$ ). 
Figura 7. Drug release from apoferritin NPs. Values represent the mean of at least 3 experiments. Standard deviation is reported in brakects.

\section{Conclusion}

Apoferritin is one of the most investigated classes of proteins applicable in drug delivery. Notwithstanding, only few studies investigated the ability of this nanocarrier in loading drugs of different activity than anticancer agents. In this paper, we demonstrated that the interaction between streptomycin sulfate and apoferritin obtained by changing $\mathrm{pH}$ values of preparation environment can lead to the formation of possible biocompatible drug delivery systems for the drug release of antimicrobial drugs as Streptomycin. The chemico-physical and technological characterization of ApoStrep NPs provided information about the operative parameters that should be set to stabilize and to modulate drug release, demonstrating that an usefull reassembly of apoferritin is greatly dependent both on the $\mathrm{pH}$ and the concentration of drug in solution. Only combining dialyses and gel filtration chromatography ApoStrep NPs were efficiently purified from unencapsulated drug and let free from aggregate; the majority of the entrapped drug molecules remained within cage (even with the stress of these purification procedures) with a good control on drug released. These preliminary data can be used for the design of new antrimicrobial delivery systems and can be also taken into account for the prediction of drug interactions within pharmaceutical formulations.

\section{Application of Apoferritin nanocages}

Despite extensive promises, much work is needed before clinical translation of APO as nanocages. As example, the study of the architecture and the structure of loaded APO are still at early stages and this situation represents the major limitation in translatability of APO to become DDS. This fact is principally due to unclear protocols for APO formulation and poor chemico-physical, morphological and technological characterization, frequently generating conflicting outputs. Regarding Streptomycin application, the dose normally used in therapy is very high, and it would be impossible to reach such doses with APO nanocages strategy. On the other hand, these systems are not designed for this scope. In fact, the possibility of exploiting nanocages able to be "safe" when circulating in the bloodstream, to slowly release the loaded drug and in a controlled manner and, most of all, to be engineered for a selective delivery should be considered as an added value and the real potential of these systems. In this work, we also tried to answer to the general lack of characterization of these systems, as we firstly analyzed the possibility of formulating an active agent different from the "commonly used" agents for loading into APO (namely anticancer drugs); then, we moved on analyzing the nature and narrow size of APO complex using analytical technique commonly used to characterize nanomedicine as liposomes or nanoparticles (TEM and PCS) and applying these technologies to APO cages characterization. Aiming to improve the chances of translatability of APO as drug delivery systems, another point, which is very hard to be investigated, is related to the drug release and in particular to the changes in morphology and architecture of APO cage during and after the release of the drugs. To our knowledge, the technologies for this kind of characterization are still lacking, even if this focus strongly affects the biocompatibility of APO and the efficacy of treatment promoted by the released drug. A huge work should therefore be done to better investigate on these aspects, that need to be considered with a major interest. 


\section{References}

Zumla, A., Nahid, P., Cole, S.T., 2013. Advances in the development of new tuberculosis drugs and treatment regimens. Nat Rev Drug Discov. 12, 388-404.

Coessens, V., Schacht, E., Domurado, D., 1996. Synthesis of polyglutamine and dextran conjugates of streptomycin with an acidsensitive drug-carrier linkage. J Control Release 38, 141-150.

Pfuetze, K.H., Ashe, W.M., 1948. Present Status of Streptomycin In Tuberculosis. Chest. 14, 446-455.

Smith, J.P., 2011. Nanoparticle Delivery of Anti-Tuberculosis Chemotherapy as a Potential Mediator Against Drug-Resistant Tuberculosis. Yale J Biol Med . 84, 361-369.

Asadi, A., 2014. Streptomycin-loaded PLGA-alginate nanoparticles: preparation, characterization, and assessment. Appl Nanosci. 4, 455-460.

Arosio, P., Levi , S., Santambrogio, P., Cozzi, A., Luzzago, A., Cesareni, G., Albertini, A., 1991. Structural and functional studies of human ferritin $H$ and $L$ chains. Curr Stud Hematol Blood Transfus. 58, $127-31$.

Burdo, J. R., Connor, J. R., 2003. Brain iron uptake and homeostatic mechanisms: An overview. BioMetals 16, 63-75.

Aisen, P. , 1991. Ferritin receptors and the role of ferritin in iron transport. Targeted Diagn Ther.4, 339-54

Maham, A., Tang, Z., Wu, H., Wang, J., Lin, Y., 2009. Protein-Based Nanomedicine Platforms for Drug Delivery. Small 5, 1706-1721.

Lin , X., Xie, J., Niu, G., Zhang, F., Gao, H., Yang, M., Quan, Q., Aronova, M.A., Zhang, G., Lee, S., , Leapman, R., Chen, X., 2011. Chimeric Ferritin Nanocages for Multiple Function Loading and Multimodal Imaging. Nano. Lett. 11, 814-819.

Lin, X., Xie, J., Zhu, L., Lee, S., Niu, G., Ma, Y., Kim, K., Chen, X., 2011. Hybrid Ferritin Nanoparticles as Activatable Probes for Tumor Imaging. Angew Chem Int Ed Engl. 50, 15691572.

Uchida, M., Flenniken, M.L., Allen, M., Willits, D.A., Crowley, B.E., Brumfield, S., Willis, A.F., Jackiw, L., Jutila, M., Young, M.J., Douglas, T., 2006. Targeting of cancer cells with ferrimagnetic ferritin cage nanoparticles. J Am Chem Soc. 128:16626-16633.

Dominguez-Vera, J.M., 2004. Iron(lii) Complexation of Desferrioxamine B Encapsulated in Apoferritin. J Inorg Biochem. 98, 469-472. 
Nys, P.S., Savitskaya, E.M., Kolygina, T.S., 1971. Determination of ionization constants of streptomycin by the indicator method. Pharm Chem J . 5, 576-579.

Belletti, D., Pederzoli, F., Forni, F., Vandelli, M.A., Tosi, G., Ruozi, B., 2017. Protein cage nanostructure as drug delivery system: magnifying glass on apoferritin, Expert opin Drug Deliv., Epub ahead of print, doi: 10.1080/17425247.2017.1243528.

Xing, R., Wang, X., Zhang, C., Zhang, Y., Wang, Q., Yang, Z., Guo, Z.,2009. Characterization and cellular uptake of platinum anticancer drugs encapsulated in apoferritin. J Inorg Biochem. 103, 1039-44.

Cutrin, J.C., Crich, S.G., Burghelea, D., Dastrù, W., Aime, S., 2013. Curcumin/Gd Loaded Apoferritin: A Novel "Theranostic" Agent To Prevent Hepatocellular Damage in Toxic Induced Acute Hepatitis. Mol Pharm. 10, 2079-2085.

Blazkova, I., Nguyen, H.V., Dostalova, S., Kopel, P., Stanisavljevic , M., Vaculovicova, M., Stiborova, M., Eckschlager, T., Kizek, R., Adam, V., 2013. Apoferritin Modified Magnetic Particles as Doxorubicin Carriers for Anticancer Drug Delivery. Int J Mol Sci. 14, 13391-13402.

Liu, G., Wang, J., Wu, H., Lin, Y., 2006. Versatile apoferritin nanoparticle labels for assay of protein. Anal Chem. 78, 7417-23.

Kilic, M.,A., Ozlu , E., Calis, S., 2012. A novel protein-based anticancer drug encapsulating nanosphere: apoferritin-doxorubicin complex.. J Biomed Nanotechnol. 8, 508-14.

Xing, R., Wang, X., Zhang ,C., Zhang, Y., Wang, Q., Yang, Z., Guo, Z., 2009. Characterization and cellular uptake of platinum anticancer drugs encapsulated in apoferritin. I Inorg Biochem.. 103, 1039-44.

Stoscheck, C.M.,1990. Quantitation of protein . Methods Enzymol. 182, 50-68

Oswald, E.J., Nielsen, J.K., 1947. Studies on the Stability of Streptomycin in Solution. Science $105,184-5$

Weisenberg, R.C., Timasheff, S.N., 1970. Aggregation of microtubule subunit protein. Effects of divalent cations, colchicine, and vinblastine. Biochemistry 9, 4110-4116.

Massover, W.H., 1993. Ultrastructure of ferritin and apoferritin: A review. Micron 24, 389-437. 
Table 1: loading capacity and encapsulation efficiency (EE) of ApoStrep D and ApoStrep DG samples

\begin{tabular}{|l|l|l|}
\hline Samples & $\begin{array}{l}\text { mg Strep/100 } \\
\text { mg of Apo }\end{array}$ & EE\% \\
\hline ApoStrep 1:10-D & $0.67 \pm 0.01$ & $44 \pm 5$ \\
\hline $\begin{array}{l}\text { ApoStrep 1:10- } \\
\text { DG }\end{array}$ & $0.53 \pm 0.01$ & $35 \pm 4$ \\
\hline ApoStrep 1:25-D & $1.27 \pm 0.02$ & $33 \pm 3$ \\
\hline $\begin{array}{l}\text { ApoStrep 1:25- } \\
\text { DG }\end{array}$ & $1.07 \pm 0.01$ & $28 \pm 1$ \\
\hline
\end{tabular}




\begin{tabular}{|l|l|l|}
\hline Sample Name & $\begin{array}{l}\text { Streptomycin concentration } \\
(\mathrm{mg} / \mathrm{mL})\end{array}$ & Apoferritin amount $(\mathrm{mg} / \mathrm{mL})$ \\
\hline Streptomycin free solution & $0.05 \mathrm{mg} / \mathrm{mL}$ & $\backslash$ \\
\hline Apoferritin solution & $\backslash$ & $15 \mathrm{mg} / \mathrm{mL}$ \\
\hline ApoStrep 1:10-DG & See table I & $10,3( \pm 0,2) \mathrm{mg} / \mathrm{mL}$ \\
\hline ApoStrep 1:25-DG & See table I & $10,1( \pm 0,3) \mathrm{mg} / \mathrm{mL}$ \\
\hline ApoStrep 1:50-DG & See table I & $2,9( \pm 0,2) \mathrm{mg} / \mathrm{mL}$ \\
\hline & & \\
\hline
\end{tabular}

Table II: concentration of Apoferritin and Streptomycin. For Streptomycin content, please see Table I. 

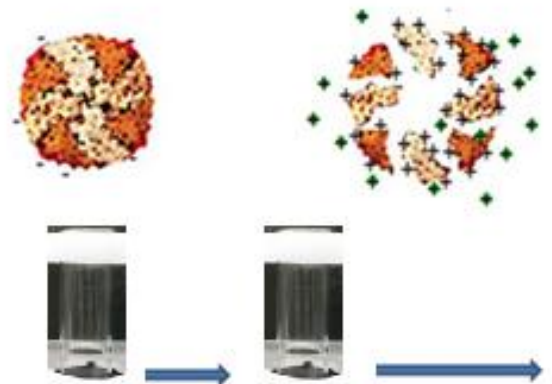

m $\mathrm{pH} 2$

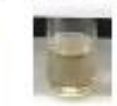

Streptomycin Sulphate
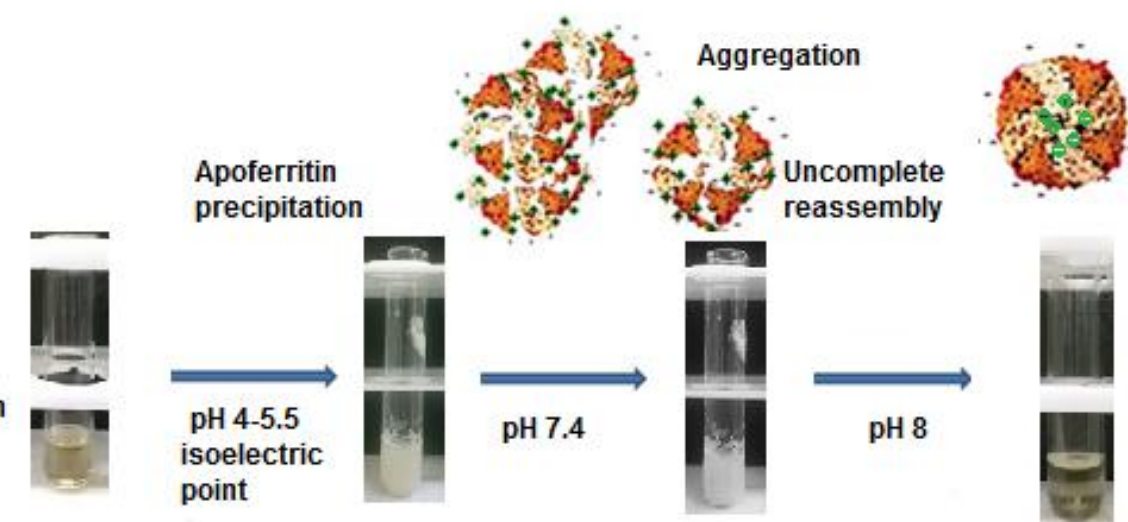

point

Figure 1. A) Schematic illustration of the encapsulation of streptomycin into apoferritin nanocage by disassembly/reassembly process via " $\mathrm{pH}$ changing method". Turbidity observed from $\mathrm{pH} 4$ to 8 is possible due to a) protein precipitation (isoelectric point at $\mathrm{pH} 4.5$ ); b) protein aggregation induced by multivalent cations as streptomycin sulfate at $\mathrm{pH} 7.4$; c) drug that could impact on the correct protein reassembling. 
Figure 2

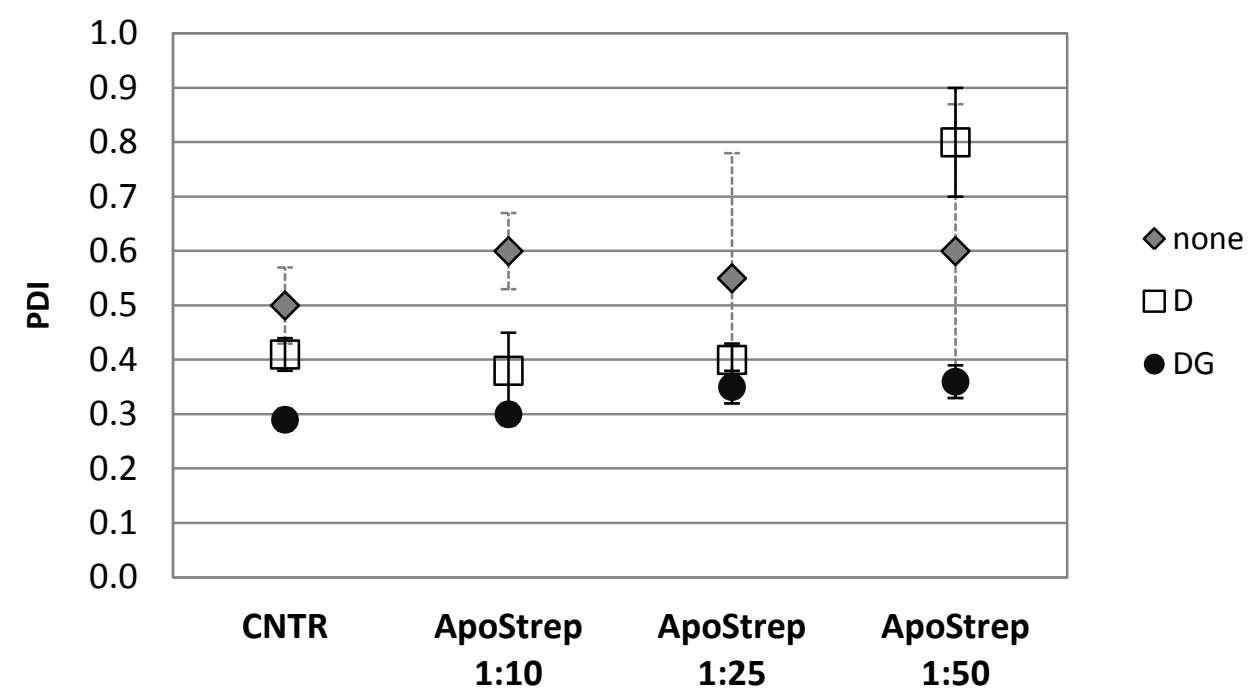

Figure 2. PDI values of apoferritin NPS (CNTR, ApoStrep 1:10, ApoStrep 1:25, ApoStrep 1:50) after $\checkmark$ formulation, $\square$ dialysis process (D) and combining dialysis and gel filtration chromatography. 

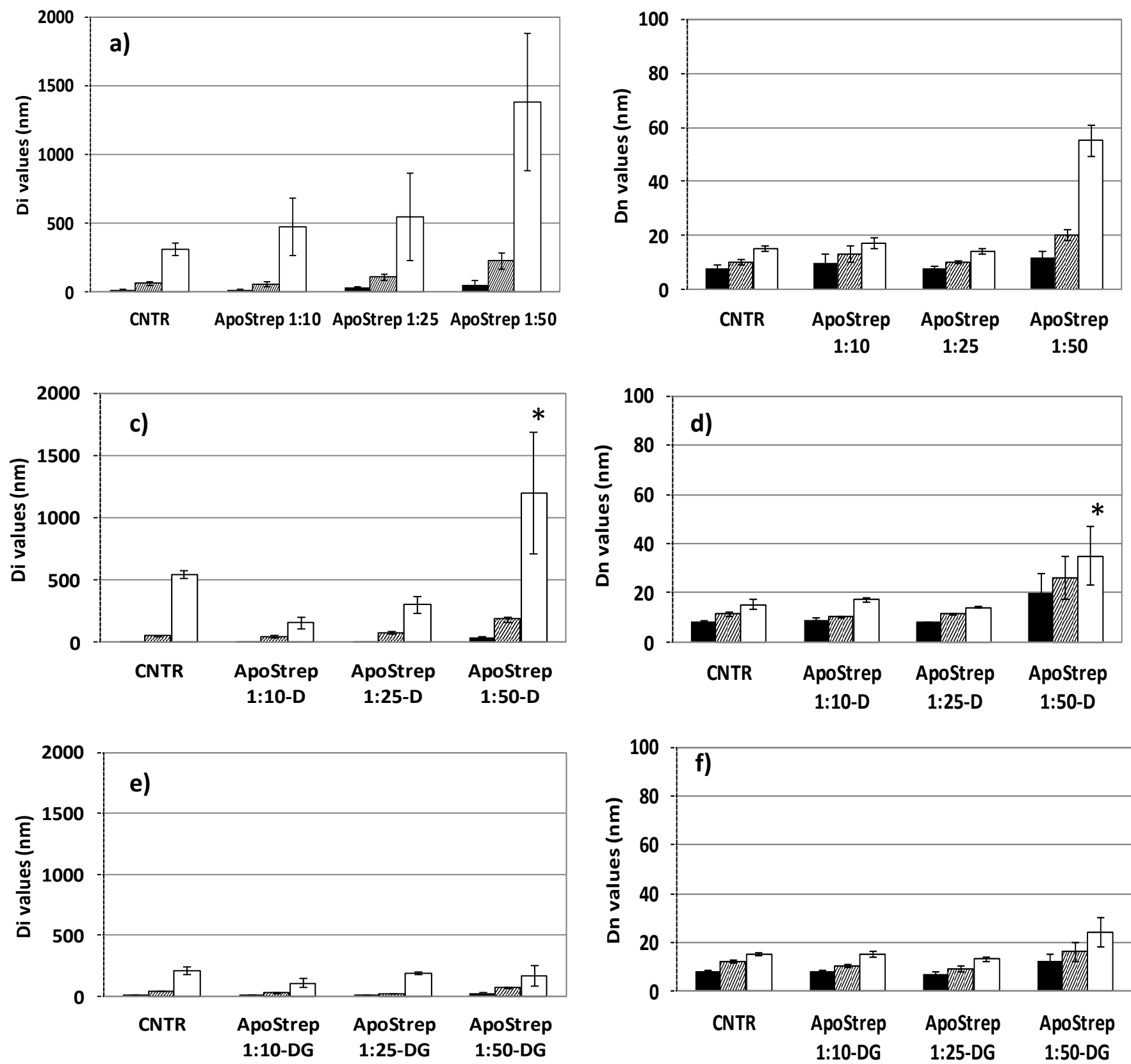

Figure 3. Dimensional characterization of ApoStrep NPs. Di (panels $a, c$ and e) and Dn (panels $b, d$ and f) of samples after formulation ( $a$ and $b$ ), dialysis ( $c$ and $d$ ), and combining dialysis and gel filtration chromatography (e and f). $\square D(10) \quad \square \quad D(50) \square \quad D(90)$. Data were analyzed using a one-way ANOVA coupled with Bonferroni post hoc test. All values are expressed as mean $\pm S E M$. ${ }^{*} P<0.05$ was the required statistical significance (vs CNTR). 


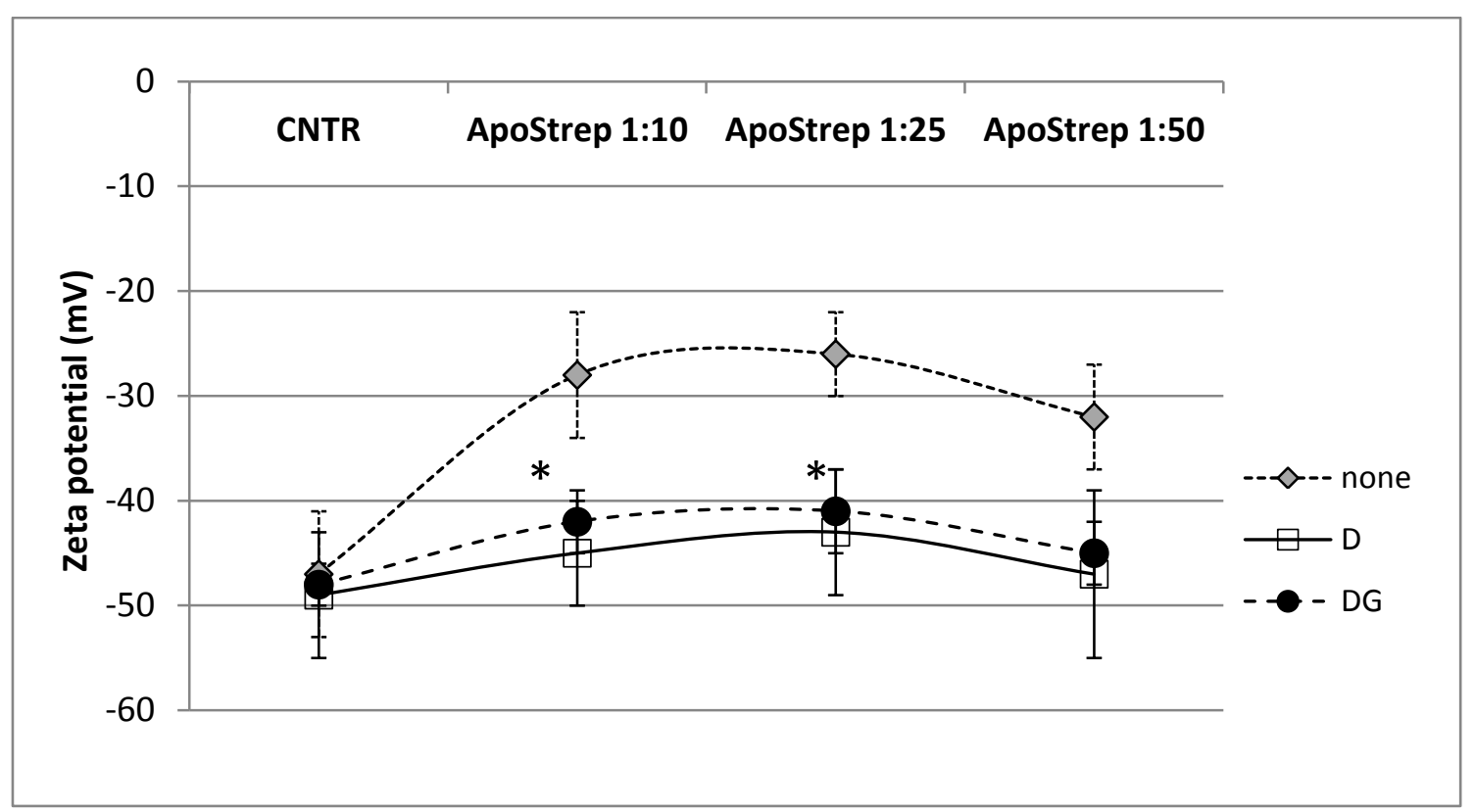

Figure 4. Zeta potential of apoferritin NPs (CNTR, ApoStrep 1:10, ApoStrep 1:25, ApoStrep 1:50) after $\checkmark$ formulation, $\square$ dialysis process (D) and combining dialysis and gel filtration chromatography. Data were analyzed using a one-way ANOVA coupled with Bonferroni post hoc test. All values are expressed as mean \pm SEM. ${ }^{*} P<0.05$ was the required statistical significance (vs "none") 
Figure 5

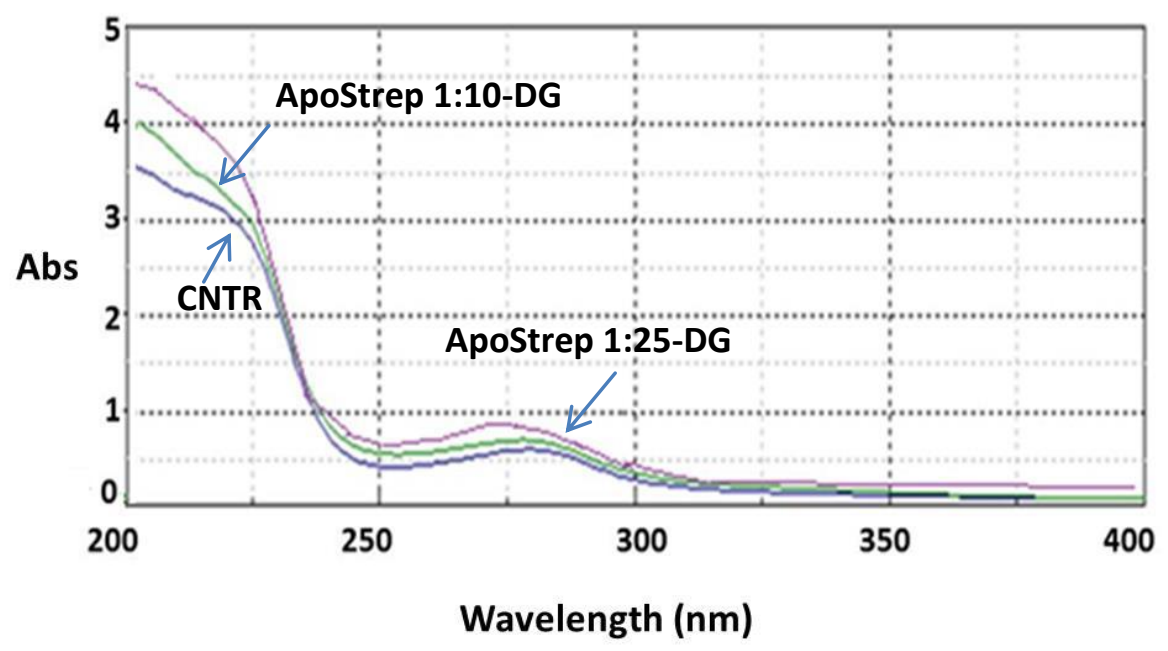

Figure 5. UV spectra of CNTR-DG vs ApoStrep1:10-DG and ApoStrep1:25-DG 
Figure 6
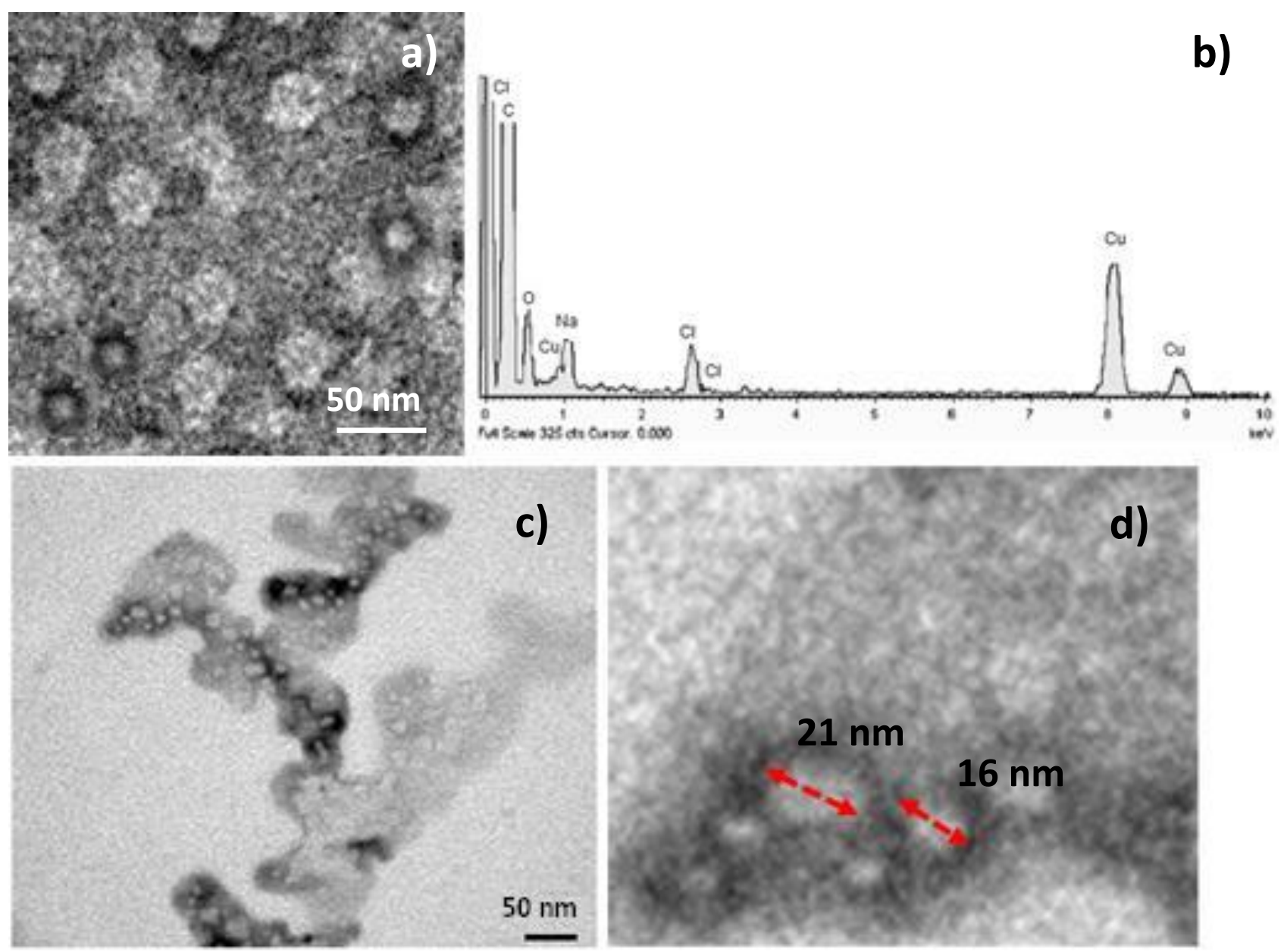

Figure 6. TEM analysis of apoferritin NPs a)Images of CNTR-DG, b)EDX of CNTR-DG, c)images of ApoStrep 1:25-DG, d) magnification of ApoStrep 1:25-DG. 


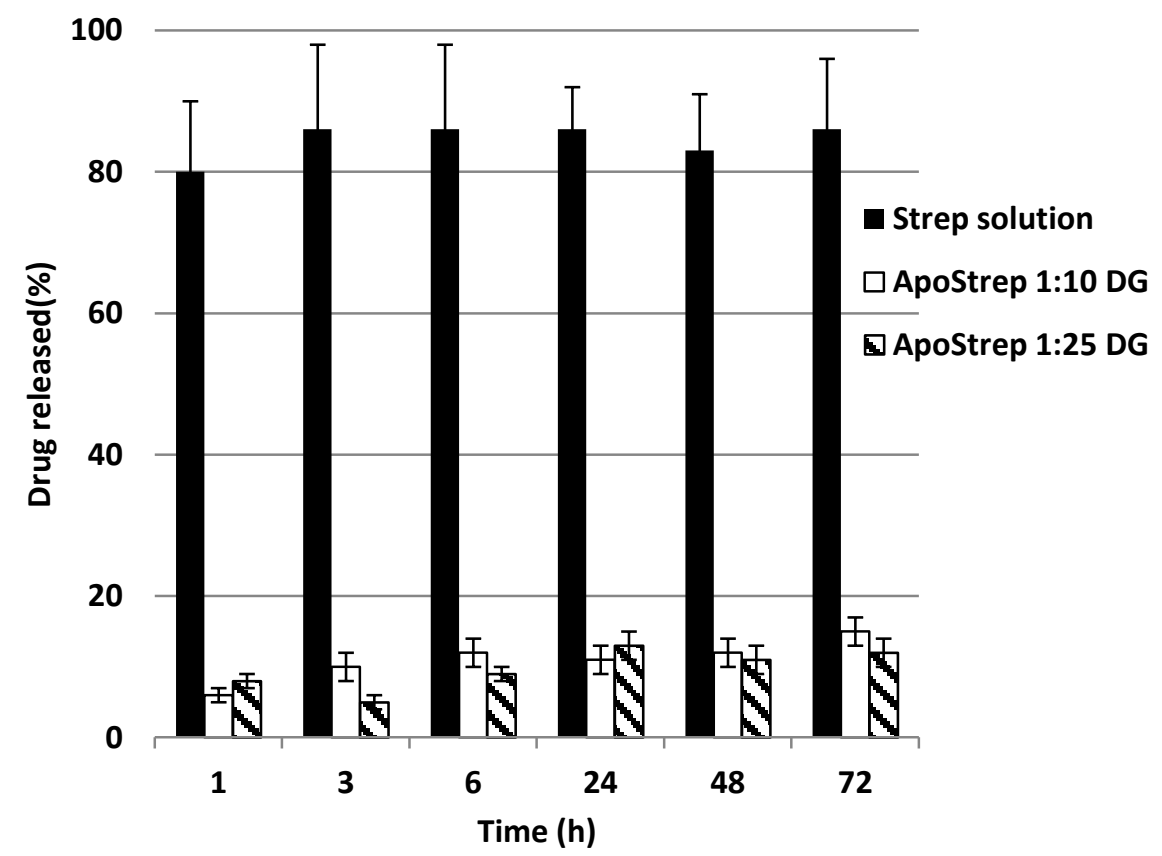

Figura 7. Drug released from apoferritin NPs. Values represent the mean of at least 3 experiments. Standard deviation is reported in brakects. 